\title{
Les compétences comme cadre pour organiser des enseignements de mathématiques ? Oui, mais ...
}

Dans plusieurs pays, les programmes scolaires sont désormais structurés en fonction de ce qu'on appelle la mouvance des compétences. Cet article illustre, parmi d'autres, une dérive potentielle de cette mouvance pédagogique, à partir de quelques observations faites en Belgique, mais à propos desquelles est faite l'hypothèse qu'elles se manifestent ailleurs et ce, quelle que soit - ou presque - la forme spécifique que prend ici ou là cette réforme des compétences. Cette dérive concerne l'usage abusif qui est fait du concept de situationproblème aux antipodes du concept de situation adidactique.

In many countries, school curricula are based on the pedagogical approach called " of competences». This paper illustrates one possible leeway observed in Belgium but probably true in other countries in despite of the form of this reform. This leeway consists in some misconception related to the concept of problem-situation far from the concept of adidactical situation.

Maggy Schneider, Professeur à l'Université de Liège, Département de Mathématique et Ladimath FUNDP-ULg, Institut de mathématique Bât. B37, 12 Grande Traverse, B-4000 Liège

Adresse électronique :

mschneider@ulg.ac.be 


\title{
Les compétences comme cadre pour organiser des enseignements de mathématiques ? Oui, mais ... Quelques dérives possibles
}

\author{
M. Schneider
}

\begin{abstract}
Dans plusieurs pays, les programmes scolaires sont désormais structurés en fonction de ce qu'on appelle la mouvance des compétences. Mon intention n'est pas ici d'analyser l'origine ni le bien-fondé de cette réforme d'enseignement que plusieurs chercheurs situent à tort ou à raison, d'une part dans le monde professionnel où le concept «d'intelligence situationnelle » fait florès et, d'autre part, dans des théories d'apprentissage socio-constructivistes. Je ne reviendrai pas non plus sur la diversité des acceptions du concept de compétence, mise en évidence par plusieurs chercheurs (e.a. P. Jonnaert, 2003), concept qui renvoie aussi bien à des capacités formelles (telle que la résolution de problèmes) qu'à des compétences plus spécifiques s'exerçant sur des objets de savoir dont on tente d'identifier les caractères épistémologiques, quand ce n'est pas à des «dispositions de nature cognitive, affective, réflexive et contextuelle»(R. Pallascio, 2000). Je me contenterai d'illustrer une dérive potentielle de cette mouvance pédagogique, à partir de quelques observations faites en Belgique, mais à propos desquelles je fais l'hypothèse qu'elle se manifeste ailleurs et ce, quelle que soit - ou presque - la forme spécifique que prend ici ou là cette réforme des compétences. Cette dérive concerne l'usage abusif qui est fait du concept de situationproblème.
\end{abstract}

\section{Problèmes et situations-problèmes}

Les trois compétences du programme québécois du secondaire en mathématique sont Résoudre une situation-problème, Déployer un raisonnement mathématique et Communiquer à l'aide du langage mathématique. Attardons-nous à la première. Une analyse du texte complet du programme fait apparaître que la résolution d'une situation-problème y est envisagée sous deux angles : "D'une part, elle est considérée comme un processus, d'où la compétence Résoudre une situation-problème. D'autre part, en tant que modalité pédagogique, elle soutient la plupart des démarches d'apprentissage de la discipline. Elle revêt une importance toute particulière du fait que le traitement des concepts mathématiques nécessite un raisonnement logique appliqué à des situations-problèmes. ». En Belgique, on désignera ces deux facettes par des expressions distinctes, en parlant de problème pour l'une et, pour l'autre, de situation-problème ou situation d'apprentissage, activité, mise en situation, ... Cependant, on retrouve dans les programmes ou ouvrages pédagogiques belges l'idée que les situations-problèmes offrent l'occasion d'une évaluation formative à la résolution de problèmes. Je reviendrai plus loin sur cette articulation mais je voudrais montrer auparavant que tout le monde ne met pas la même chose derrière le concept de situationproblème.

\section{Situations-problèmes et situations adidactiques}

Le concept de situation-problème souffre d'une polysémie implicite questionnée par plusieurs chercheurs dont R. Pallascio (2005), à propos de l'enseignement fondamental au Québec: "Il existe un certain niveau de confusion concernant le concept de situationproblème, qui apparaît dans plus d'une discipline du Programme de formation de l'école 
québécoise (MEQ 2001), dans la manière de l'interpréter, en confondant, par exemple, projet et situation-problème. Y a-t-il une différence entre un "simple » problème ayant pour objet de contextualiser une notion mathématique et une situation-problème mathématique à résoudre ?». Pour percevoir cette polysémie, il est bon de revenir aux sources.

On situe souvent - cf. e.a. M. Fabre, 1999 - l'origine du développement scolaire des situations-problèmes dans les travaux de didactique des mathématiques, en particulier ceux de G. Brousseau (1998). Dans ces derniers, le concept de situation adidactique - auquel renvoie la notion vulgarisée de situation-problème - fait partie d'un réseau conceptuel qui lui confère sa portée et sa consistance épistémologiques et se définit par plusieurs caractéristiques dont nous en retiendrons quatre pour notre présent propos.

1. Une première caractéristique met en jeu la connaissance mathématique visée comme "seul moyen» ou, du moins, comme savoir optimal permettant de résoudre la question mobilisée dans la situation adidactique et, plus généralement, la classe de problèmes d'où cette question est issue. D'où le lien avec la notion de situation fondamentale que G. Brousseau (1998) décrit initialement en ces termes : "Chaque connaissance peut se caractériser par une (ou des) situation adidactique qui en préserve le sens et que nous appellerons situation fondamentale».

2. Une deuxième caractéristique d'une situation adidactique est qu'elle est susceptible d'être dévolue aux élèves, ce qui suppose l'existence d'un milieu permettant à l'élève de se situer sans se référer aux attentes supposées du professeur. L'écueil majeur étant ce qu'on peut appeler les effets pervers du contrat didactique qui feraient prisonniers, tant le professeur que l'élève, d'un jeu de dupes dans lequel chacun des deux « négocierait » à la baisse le comportement attendu de l'autre.

3. Une troisième caractéristique, conséquence des deux premières, est qu'une situation adidactique se doit de déboucher sur une phase d'institutionnalisation au cours de laquelle le professeur identifie, dans les activités de l'élève, "celles qui ont un intérêt, un statut culturel ». Le savoir apparaît alors comme réponse socialement construite en réponse à une classe de problèmes dont la situation adidactique constituait un ou, mieux, plusieurs exemples prototypiques. Le processus d'institutionnalisation est donc bien l'indice qu'un enjeu de savoir est visé par la (les) situation adidactique.

4. Enfin, une quatrième caractéristique sera importante pour notre analyse ultérieure. Cette caractéristique, qui peut indûment apparaître de pure forme, a trait au partage du temps et des responsabilités au sein de la classe. Traditionnellement, le professeur expose d'abord la théorie et les élèves résolvent ensuite les exercices et les problèmes d'application de cette théorie. Dans une première rencontre avec le savoir basée sur un jeu de situations adidactiques, on voit De l'extérieur un temps partagé d'une toute autre manière: les élèves sont «mis en activité» dans un premier temps et le professeur expose ensuite. Cependant, au-delà de cette seule observation, une situation adidactique suppose un réel partage des responsabilités, dans le sens où les élèves participent, de par leurs productions, à la progression du cours.

Ainsi que le montrent M. Schneider et A. Mercier (à paraître), plusieurs exemples de situations-problèmes sont loin de satisfaire les caractéristiques d'une situation adidactique et ce, même au sein des programmes scolaires ou d'institutions de recherche. Voici deux de 
leurs exemples choisis dans des documents belges qui s'affichent comme relevant de l'idéologie des compétences.

Exemple 1 Un premier exemple est extrait d'un programme scolaire (FESeC, 2000), assorti d'illustrations de «situations d'apprentissage ». Il s'agit ici d'amener les élèves à formuler le cas de similitude de deux triangles qui se base sur l'égalité de deux angles respectifs. Les enjeux de la situation d'apprentissage sont rédigés ainsi : "Etablir que deux triangles qui ont seulement deux angles de même amplitude sont semblables, dans une configuration qui induit de conjuguer une rotation et une homothétie. Analyser ainsi un modèle de croissance tel qu'on le rencontre dans la nature, par exemple dans le cas de la coquille de l'argonaute ».

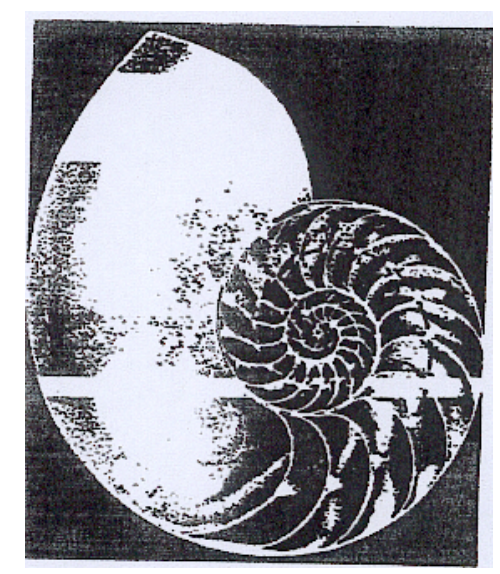

Fig. 1

Et voici l'énoncé de l'activité : Diviser l'angle de $360^{\circ}$ en cinq parties de même amplitude par une étoile de demi-droites issues du sommet $O$ de cet angle. Choisir un point $P$ sur une de ces demi-droites. Tracer à partir de $P$ et jusqu'à la demi-droite suivante dans un sens à choisir, un segment [PQ] faisant un angle de $65^{\circ}$ avec la demi-droite [OP. Recommencer la même construction dans le même sens à partir du point $Q$ et ainsi de suite. Comparer de proche en proche les différents triangles qui forment cette spirale. Le déroulement est prévu par les auteurs : "Chaque élève construit une spirale sur une feuille blanche de format A3. Pour pouvoir tracer six triangles, il est recommandé de ne pas choisir $P$ trop éloigné de $O$. Il est en effet nécessaire de tracer six segments pour voir apparaître une situation de Thalès et avoir ainsi l'intuition qu'il existe des rapports égaux entre côtés. Pour comparer deux triangles 


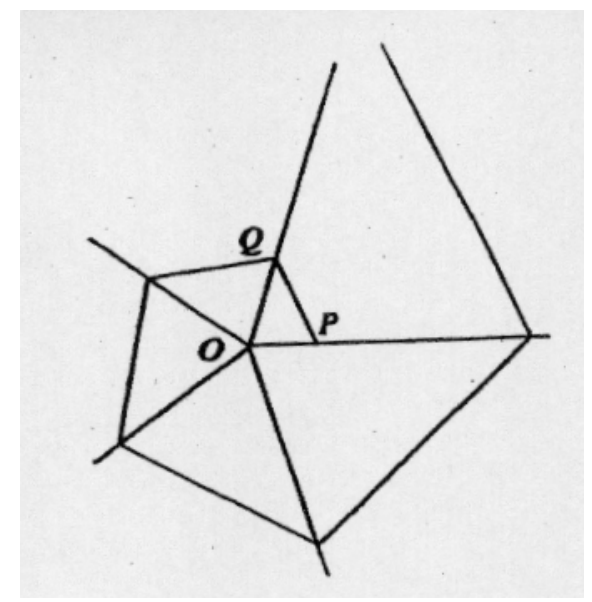

Fig. 2

consécutifs on opère une rotation de $72^{\circ}$ qui envoie le premier triangle à l'intérieur du second, le second à l'intérieur du troisième ... Le mieux est de reproduire la spirale sur papier transparent : la rotation s'opère d'un seul mouvement (voir Fig. 2). »

Dans cet exemple, il y a bien un enjeu de savoir mathématique : la formulation d'un cas de similitude. Cependant, ce savoir est loin d'être optimal pour répondre à la consigne ; comme le disent les auteurs eux-mêmes, l'énoncé induit de faire appel aux transformations du plan grâce auxquelles on déplace un triangle dans l'autre pour faire apparaître un autre mode de comparaison des triangles et même une autre forme de validation de leur similitude par le biais d'un savoir ancien : le théorème de Thalès. Cette brève analyse fait apparaître qu'il y a peu de chances que le savoir visé émerge spontanément et on peut imaginer les effets de contrat qui seront sans doute nécessaires pour faire «marcher» cette situation. Nous irons même plus loin en disant qu'il y a ici un milieu propice au savoir concurrent de celui visé car, si l'on se réfère aux propos de R. Bkouche (1988) ci-dessous en les extrapolant, les cas d'égalité (ou de similitude) des triangles ont pour intérêt majeur de permettre la comparaison de triangles sans que l'on ait à « déplacer » l'un par rapport à l'autre, que ce soit par référence implicite au mouvement ou par usage explicite de transformations : «Axiome fondateur de la géométrie, le principe de l'égalité par superposition s'appuie essentiellement sur la notion de mouvement; la géométrie est ainsi fondée empiriquement sur le lien entre corps solide et mouvement, et c'est la coïncidence par transport d'un corps sur un autre qui permet de conclure à l'égalité de deux corps [...]. Le problème de la géométrie est alors d'énoncer $a$ priori des conditions d'égalité, ce qui permettra d'éliminer le mouvement, remplacé par un raisonnement s'appuyant sur les critères d'égalité ainsi définis ».

Pointons, pour terminer, un autre des enjeux de cette situation annoncés par les auteurs et sur lequel je reviendrai: "Analyser un modèle de croissance tel qu'on le rencontre dans la nature » parce qu'il nous paraît paradigmatique de références très souvent invoquées pour justifier l'intérêt d'une situation-problème : dans ce rôle, on trouve aussi bien la nature que la vie courante ou les autres disciplines. Ce qui n'empêche évidemment pas que de tels contextes puissent servir de point d'appui à la construction d'une situation authentiquement adidactique. Cependant, nous regrettons que, dans un certain nombre de cas, la présence seule d'un contexte puisse permettre de labelliser « situation-problème » une activité donnée. 
Exemple 2 Un deuxième exemple est une «activité de découverte » extraite de CREM, 2004 et dont l'intégralité est reprise ci-dessous : d'abord l'activité proprement dite et ensuite une solution sommaire telle qu'attendue par les auteurs.

De quoi
a-t-on
besoin?

Comment s'y prendre?

\section{Matériel}

Une grande quantité de polygones réguliers en carton, tous à côtés égaux entre eux, et du papier collant; ou bien les mallettes de Polydron.

\section{Prérequis}

La séquence 2.

Le professeur propose aux élèves de repartir d'une tentative de pavage régulier impossible, au moyen des pentagones.

Comment faire pour assembler trois pentagones? Comment "supprimer le trou"?

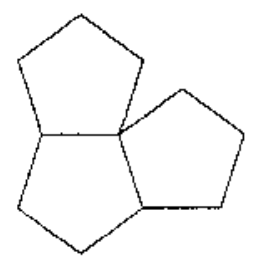

Fig. 3

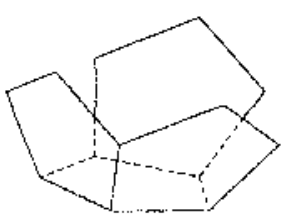

Fig. 4

La solution consiste à passer du plan à l'espace. En effet, si on relève les pentagones autour du nœud, celui-ci devient un sommet et les côtés coïncident alors parfaitement. La forme obtenue est une sorte de coupelle composée de trois pentagones autour d'un sommet. On peut alors ajouter un autre pentagone sur un des côtés (Fig. 4), et ainsi de suite. »

La question dévolue dans le cadre fait suite à des activités de pavage du plan au moyen de diverses figures géométriques. Il s'agit donc ici de faire un pas de côté par rapport à cette problématique pour penser «espace » et non plus «plan», ce qui ne semble pas aller de soi pour les élèves, comme en témoignent les auteurs rapportant des réflexions significatives telles que «Ce n'est pas un pavage car on ne travaille plus dans le plan, mais dans l'espace. » Suit une argumentation qui montre qu'il existe cinq polyèdres réguliers seulement alors qu'il y a une infinité de polygones réguliers. Elle est ponctuée de questions qui laissent peu d'initiative aux élèves et que nous n'analyserons pas ici. Notre intention se résume à questionner l'activité ci-dessus et son statut de transition entre deux problématiques : des pavages du plan aux polyèdres réguliers. Elle requière un changement de regard que rien n'induit a priori, demandant ainsi une plasticité mentale que d'aucuns reconnaissent assez rare. On sent ici l'absence d'un milieu pour dévoluer la «découverte » du savoir visé annoncé : les polyèdres de Platon, à l'instar du milieu aménagé par G. Polya (1967) ou, plus récemment, par T. Dias et V. Durand-Guerrier (2005) qui prend appui sur l'expérimentation physique du fait suivant: la somme des angles autour d'un sommet du polyèdre doit être inférieure à $360^{\circ}$.

Ce cas est assez symptomatique d'un fonctionnement au principe de la conception de certaines «situations-problèmes » ainsi que le développe M. Schneider (2002) en introduisant le concept d'obstacle psychologique pour désigner ce phénomène. Cette expression est choisie par référence aux psychologues du comportement tels P. Oléron qui, dans les années 
80, ont étudié les attitudes et habitudes mentales liées au sujet et qui font obstacle à la résolution d'un problème. Un des cas exemplaires s'apparente d'ailleurs à l'exemple décrit plus haut. Il s'agit du problème des allumettes de Duncker : construire quatre triangles équilatéraux au moyen de six allumettes. La réponse est le tétraèdre régulier mais les psychologues observent que la plupart des personnes ne s'autorisent pas à penser le problème dans l'espace se donnant la contrainte imaginaire de manipuler les allumettes dans le plan. Remarquons, qu'en l'absence de colle, on ne pouvait guère espérer un autre comportement.

Comme le montre M. Schneider (Ib.), les obstacles psychologiques semblent jugés emblématiques, sur le terrain, d'une «authentique » démarche de résolution de problèmes, celle-ci se caractérisant par une innovation permettant de résoudre une situation "inédite». Cela caractériserait aussi bien des problèmes d'application d'une théorie déjà exposée que des situations-problèmes ou, comme les appellent G. Noël et al. (2001), des problèmes d'introduction à une théorie. Et c'est bien pour cela que les situations-problèmes peuvent être conçues, par ces auteurs, comme un entraînement à la résolution de problèmes, dans le cadre d'une évaluation formative.

Cette conception gagne du terrain en particulier dans le cadre de la mouvance des compétences. Elle se cristallise dans des documents officiels tels que des documents d'accompagnement des programmes. Ainsi, dans un document FESeC (1995), les situationsproblèmes s'intègrent dans une "pédagogie de la recherche », expression significative s'il en est, du moment qu'elles satisfassent à l'une ou l'autre des caractéristiques suivantes, voire à plusieurs :

- elle constitue un défi, suscite un étonnement, crée une surprise,

- elle invite l'élève à faire quelque chose (compter - faire un dessin - calculer - couper),

- elle laisse à l'élève une certaine liberté quant au choix de sa méthode et de ses conjectures (suppositions fondées sur des apparences) et met en œuvre sa créativité,

- elle est issue du terrain de l'élève (dans le domaine de son savoir quotidien et de ses acquis scolaires),

- elle met en œuvre une réflexion qui dépasse l'utilisation immédiate de résultats antérieurs,

- elle permet de rencontrer plusieurs notions différentes,

- $\quad$ elle conduit l'élève à rédiger sa démarche, son raisonnement.

Ces caractéristiques possibles d'une situation-problème, dont on ne sait a priori quelles sont celles qui seront respectées, ne constituent pas un cadre théorique consistant en raison même du fait que leur choix autorise des exemples fort diversifiés dont certains peut-être pourraient s'apparenter aux jeux adidactiques de Brousseau mais dont d'autres leur sont totalement étrangers.

Comme on le voit sur les exemples repris ci-dessus, ce n'est pas parce qu'une activité des élèves précède le discours du professeur qu'il existe un réel partage des responsabilités, au sens décrit dans la $4^{\text {ème }}$ caractéristique des situations adidactiques, permettant que fonctionne la dévolution d'une question aux élèves, sans intervention arbitraire du professeur c'est-à-dire grâce à l'existence d'un milieu. Seules les apparences sont sauves du fait d'une organisation temporelle du cours formellement semblable à celle que suppose une situation adidactique.

De manière générale, il existe plusieurs principes organisateurs des situationsproblèmes rencontrées sur le terrain : leur caractère concret (référence à la nature, à la vie de 
tous les jours, ...), un obstacle psychologique ou encore une occasion de traduire en langage mathématique un énoncé de la langue véhiculaire, ... Ces principes ne sont pas forcément contradictoires avec les caractéristiques d'une situation adidactique, mais il me semble qu'une hiérarchisation s'impose, en cas de concurrence, si l'on ne veut pas enfermer la dévolution des situations dans un cul-de-sac.

\section{Les situations-problèmes comme occasion d'évaluation formative à la résolution de problèmes ?}

L'idée me paraît a priori caduque, bien que ce soit celle défendue par plusieurs partisans de la mouvance des compétences qui tablent sur un apprentissage progressif de l'autonomie (cf. e.a. G. Noël et al., 2001, X. Roegiers, 2000). Et ce, pour des raisons que je développe dans M. Schneider (2002 et 2006b) et dont je reprends ici quelques éléments.

Il est vrai que les démarches engagées par les élèves à l'occasion des situationsproblèmes qui leur sont proposées participent à un entraînement à la résolution de problèmes. D’une certaine façon, c'est bien le moins. Réciproquement, les démarches méthodologiques peuvent faciliter l'émergence des savoirs. Toutefois, cette vision des choses comporte des risques. Principalement, l'idée de concevoir les situations-problèmes comme entraînement à la résolution de problèmes se heurte à un écueil lié à la nécessaire "dépersonnalisation "que suppose une bonne articulation des processus de dévolution et d'institutionnalisation des situations adidactiques : il importe que l'élève se polarise sur le savoir mis en jeu et son efficacité à résoudre le problème, quelle que soit la personne qui propose ce savoir: luimême, un de ses pairs ou, pourquoi pas, le professeur. A contrario, avoir conscience que la situation-problème lui sert de piste d'essai pour s'entraîner à la résolution de futurs problèmes inédits risque de recentrer les préoccupations de l'élève sur sa propre créativité ou sur la pertinence de ses choix à lui, et ainsi de “ repersonnaliser" le jeu. Rien de tel n'est voulu $a$ priori dans un enseignement organisé à partir de situations adidactiques qui se conçoivent dans une démarche collective de mise à l'épreuve de savoirs et dont les ruptures de contrat associées doivent être équilibrées par un maintien très fort de la relation didactique. En outre, contrat didactique oblige, les élèves doivent pouvoir croire que la capacité à résoudre des problèmes est moins tributaire de leur ingéniosité personnelle que de leurs connaissances.

Me basant sur des résultats de recherche tant en psychologie cognitive qu'en didactique des mathématiques, j'ai proposé un modèle d'apprentissage de la résolution de problèmes (M. Schneider, 2002 et 2006b) et que je formule schématiquement comme suit, à la lumière des «praxéologies » d'Y. Chevallard (1999). Des questions relevant d'une même problématique seraient exposées d'entrée de jeu aux élèves; elles leur seraient ensuite dévolues pourvu qu'elles aient pu se traduire en situations adidactiques, ou, à défaut, explorées par le professeur devant les élèves par le biais d'un discours métacognitif portant sur le savoir (auquel cas, on ne parlera évidemment pas de situation-problème, ... et l'objet principal de la dévolution sera l'exploration de la technique dans un champ de problèmes parents). De cet examen qui ferait ressortir l'essence commune de ces questions devrait émerger une technique type de résolution. Les questions seraient alors cristallisées en une classe de problèmes et le discours technologique qui valide cette "technique" (au sens large du terme) déboucherait sur un embryon (ou un pan de théorie), lequel institutionnaliserait la technique comme répondant à cette classe de problèmes. Les élèves seraient alors entraînés à la résolution de problèmes de cette classe et invités à explorer le domaine d'opérationnalité de la technique de résolution jusqu'à en éprouver les limites. Ils seraient enfin évalués sur leur capacité à transférer la méthode de résolution à de nouveaux problèmes de la même classe. 
Dans une telle perspective, les savoirs construits outillent les élèves pour résoudre une classe particulière de problèmes, puis une autre et ainsi de proche en proche de sorte qu'ils disposent d'un arsenal de connaissances leur permettant de faire face à un nombre sans cesse croissant de types de problèmes. Mais peut-on exercer et évaluer la compétence " résolution de problèmes" dans le scénario décrit ci-dessus, objecteront certains. De fait, à force de faire explorer aux élèves le domaine de validité d'une technique de résolution associée à une classe de problèmes, on ne peut guère, au terme de l'apprentissage en cours, que tester leur capacité à exploiter cette même technique pour résoudre un problème qu'ils identifient d'office, contrat didactique oblige, comme faisant partie de la classe étudiée. Cependant, un enjeu de transfert non négligeable se profile dès que l'élève, susceptible de maîtriser plusieurs classes de problèmes, doit reconnaître, par analyse des invariants et des spécificités, à quelle classe appartient tel ou tel problème qui lui est proposé, tout comme un expert le ferait d'ailleurs. D'où l'intérêt de proposer des évaluations où, de manière affichée et effective, différentes classes de problèmes sont brassées d'une année à l'autre, afin d'éviter les effets de contrat poussant l'élève à adopter telle méthode ou telle autre en fonction des contenus de programmes travaillés pendant l'année en cours.

\section{Le risque d'une implosion didactique}

S'il est temps de proposer un tel modèle d'apprentissage de la résolution de problèmes, c'est en raison d'un engouement aveugle pour le «transversal » porté par la mouvance des compétences et qui se traduit, en mathématiques, par l'illusion d'un apprentissage à la démarche de résolution de problèmes pensé en marge des apprentissages de savoirs. Dans $\mathrm{M}$. Schneider (2006a), je relate plusieurs faits divers à l'appui de cette thèse dont le suivant. Il concerne ce que l'on appelle les «problèmes de dénombrement» au niveau des deux premières années de l'enseignement secondaire, dans les programmes scolaires belges ou dans les manuels. Ces problèmes, présents également dans les enquêtes PISA, supposent d'identifier d'abord une régularité dans une suite de nombres figurés par des objets et d'exprimer ensuite cette régularité par une formule susceptible de donner le nombre d'objets à toute étape. Des professeurs enseignant dans des écoles voisines font part à un conseiller pédagogique de leur perplexité face à ces problèmes que leurs élèves échouent souvent à résoudre. Le conseiller rassemble plusieurs de ces problèmes et explique aux professeurs, qui n'en sont pas conscients, les diverses structures fonctionnelles sous-jacentes à ces problèmes. Dans le cas présent, celles-ci sont au nombre de cinq et des techniques particulières permettent de les repérer et de les traiter au delà du contexte : ainsi, une suite arithmétique se traduit par des écarts constants d'un terme au suivant et la formule associée ne fait que rendre compte du nombre de fois qu'il faut ajouter cet écart pour obtenir un terme donné... Les professeurs apprécient l'enseignement prodigué par le conseiller pédagogique car, disent-ils, ce discours les aide eux-mêmes à y voir plus clair dans le «fouillis » des problèmes qu'ils parviennent enfin à «catégoriser». Le conseiller pédagogique les engage à travailler ces problèmes de la même façon avec leurs élèves. La réponse des professeurs est le fait que je voudrais pointer ici : «Si nous faisons la même chose avec nos élèves, ce ne sera plus pour eux de la résolution de problèmes ». On a là l'exemple de professeurs qui, pour des raisons tout à fait louables dont font partie le souci d'une évaluation «scientifique » de la résolution de problèmes et le souhait d'y préparer leurs élèves, s'interdisent tout simplement d'enseigner avec l'illusion que l'activité « autonome » des élèves en tant que telle les prépare à gérer les situations futures. 
En guise de conclusion, je soulignerais le risque d'implosion didactique que me font craindre des anecdotes semblables à la précédente. Comment S. Johsua (2002), je pense qu'il y a un «détournement de la problématique »dans la mouvance des compétences : on cherche à rendre les élèves capables de résoudre des problèmes jugés importants dans une société donnée et on les prive, en même temps, des savoirs construits culturellement pour gérer ces situations de référence. Comme je l'ai argumenté (M. Schneider, 2006a), ce détournement de la problématique conduit à une « décatégorisation » des questions étudiées à l'école, fédérées désormais non plus par les savoirs mais par des compétences transversales telles Se poser des questions, Communiquer, compétences dont on peut craindre un fonctionnement commun à plusieurs disciplines qui écrase les spécificités épistémologiques de ces dernières tout en induisant des apprentissages factices chez les élèves.

\section{BIBLIOGRAPHIE}

Brousseau, G. (1998). La théorie des situations didactiques. Grenoble : La pensée Sauvage. Chevallard, Y. (1999). L'analyse des pratiques enseignantes en théorie anthropologique du didactique. Recherches en Didactique des Mathématique, 19(2), 221-266.

CREM (2004), Pour une culture mathématique accessible à tous. Ouvrage publié avec le soutien de la Communauté française de Belgique.

Dias T. § Durand-Guerrier, V. (2005). Expérimenter pour apprendre en mathématiques. Repères-IREM, 60, 61-78.

Fabre, M. (1999), Situations-problèmes et savoir scolaire. Paris : PUF.

FESeC (1995). Document d'accompagnement du programme de Mathématiques du $1^{\text {er }}$ degré de l'enseignement secondaire, D/1995/0279/073. Bruxelles : Licap.

FESeC (2000). Programme de Mathématiques, $2^{\text {ème }}$ et $3^{\text {ème }}$ degrés, Humanités générales et technologiques, D/2000/7362/023. Bruxelles : Licap.

Johsua S. (2002). La popularité pédagogique de la notion de compétence peut-elle se comprendre comme une réponse indaptée à une difficulté didactique majeure ?. In J. Dolz $\S$ E. Ollagnier (Eds.), L'énigme de la compétence en éducation, (pp. 115-128). Bruxelles : De Boeck.

Jonnaert P. (2003). Compétences et socioconstructivisme. Bruxelles : De Boeck.

MEQ (2001). Programme de formation de l'école québécoise, enseignement secondaire. Document disponible sur le site du Ministère de L'Education, de Loisir et du Sport. htpp://www.mels.gouv.qc.ca (consulté le 30 juillet 2006)

Noël G. § Tilleuil P. § Cazzaro J.-P. § Pourbaix F. (2001). Structurer l'enseignement des mathématiques par des problèmes, 1. Mathématique et Pédagogie, 130, 39-63.

Pallascio R. (2000). Vers une contextualisation des apprentissages par compétences (document informatique définitivement indisponible).

Pallascio R. (2005). Les situations-problèmes : un concept central du nouveau programme de mathématique. Vie pédagogique, 36, 32-35.

Polya G. (1967). La découverte des mathématiques. Paris : Dunod.

Rey B. (1996). Les compétences transversales en question. Paris : ESF

Roegiers X. (2000). Une pédagogie de l'intégration. Compétences et intégration des acquis dans l'enseignement. Bruxelles : De Boeck Université.

Schneider M. (2002). Problèmes et situations-problèmes : un regard pluraliste. Mathématique et Pédagogie. 137,13-48.

Schneider M. (2004). Viser le «transversal» à travers du «bon disciplinaire» ou trois compétences transversales contextualisées au sein des mathématiques. Repères-IREM, 55, 5170 . 
Schneider M. (2006a). Quand le courant pédagogique "des compétences » empêche une structuration des enseignements autour de l'étude et de la classification de questions parentes. Revue française de Pédagogie, 154, 85-96.

Schneider M. (2006b). Comment des théories didactiques permettent-elles de penser le transfert en mathématiques ou dans d'autres disciplines?. Recherches en Didactique des Mathématiques, 26, (1), 9-38.

Schneider M. § Mercier A. (à paraître). Situation adidactique, situation didactique, situationproblème : circulation de concepts entre théorie didactique et idéologies pour l'enseignement. Actes du Colloque "Didactiques : quelles références épistémologiques? », Bordeaux, mai 2005. 